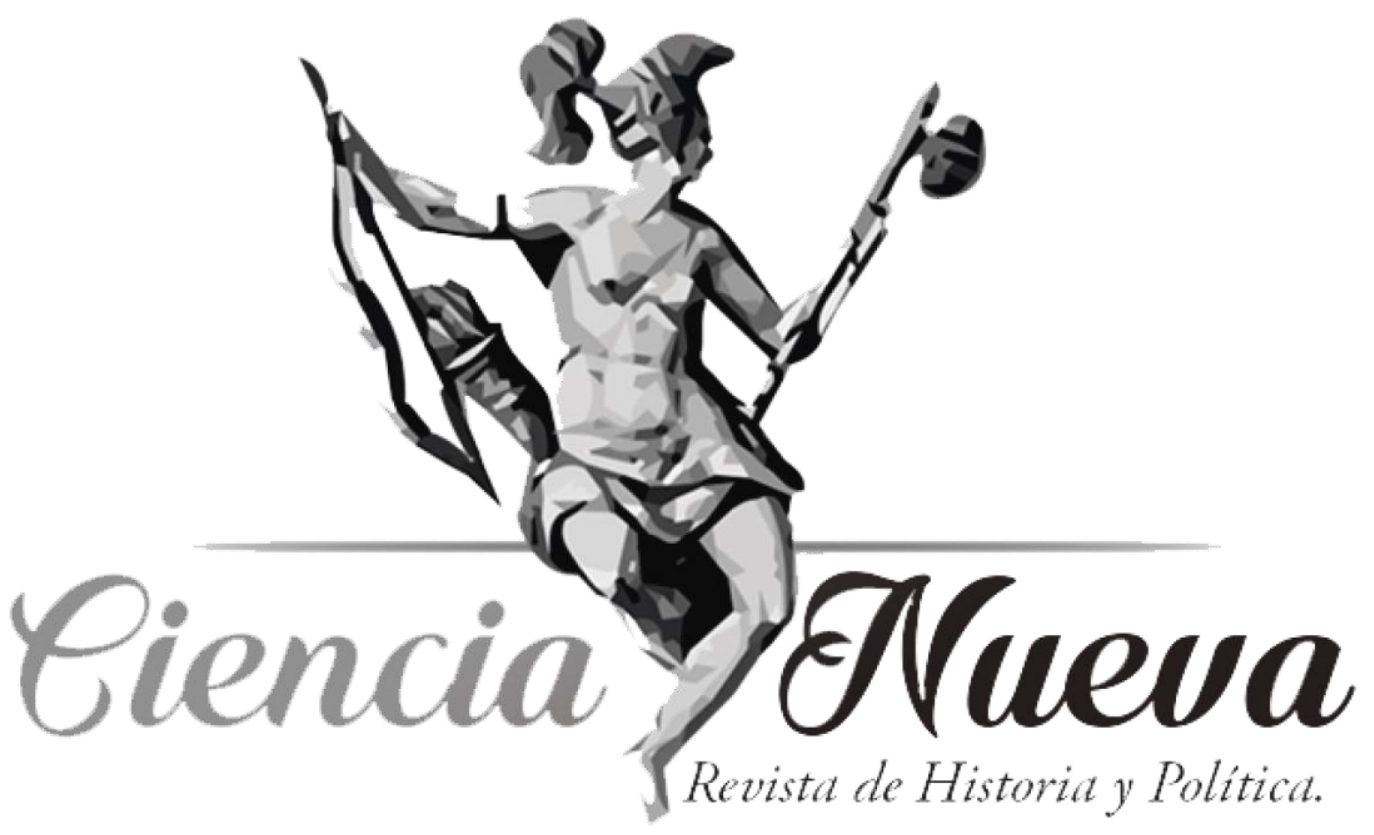

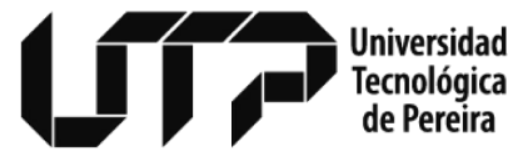

Maestría en Historia

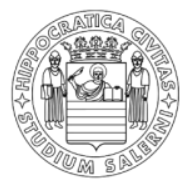

UNIVERSITÀ DEGLI STUDI DI SALERNO

Maestría en Ciencia Política

DOSSIER: ENSEÑANZA DE LA HISTORIA E HISTORIA DE LA EDUCACIÓN

\title{
LA MODERNIZACIÓN DE LA EDUCACIÓN MEDIA EN PEREIRA: EL CASO DEL INEM FELIPE PÉREZ (1969-1972)
} THE MODERNIZATION OF THE SECONDARY EDUCATION IN PEREIRA: THE CASE OF THE INEM FELIPE PÉREZ (1969-1972)

Alba Lucía Pérez Gil

pp. 102-123

Vol. 2 Núm. 2, Julio-Diciembre de 2018 Pereira, Colombia 


\section{LA MODERNIZACIÓN DE LA EDUCACIÓN MEDIA EN PEREIRA: EL CASO DEL INEM FELIPE PÉREZ (1969-1972)* THE MODERNIZATION OF THE SECONDARY EDUCATION IN PEREIRA: THE CASE OF THE INEM FELIPE PÉREZ (1969-1972)}

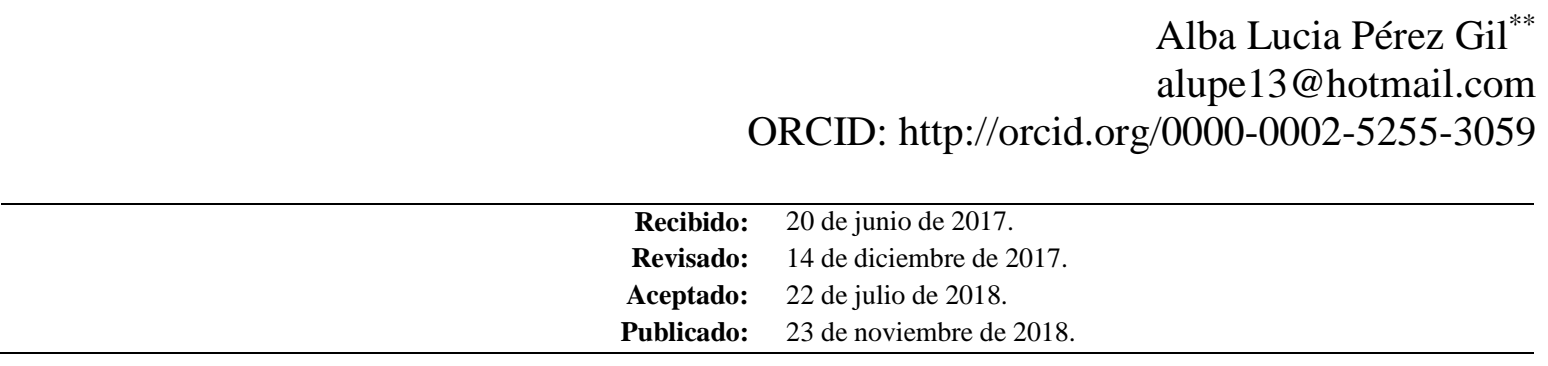

\section{Resumen}

El artículo presenta un acercamiento al proceso de modernización de la educación media en Pereira. El sistema INEM fue una expresión de la modernización educativa vivida en el país a partir de la segunda mitad del siglo XX. Según Martínez Boom, se desarrolló en dos momentos: escuela expansiva y escuela competitiva. El periodo comprendido entre 1950 y 1980 corresponde a la primera fase de la modernización (escuela expansiva). Se fundamentó en el modelo de desarrollo en términos de crecimiento económico, el cual demanda de la escuela expansión. Esta primera fase es entendida como el ciclo cuantitativo de la escuela para extender la escolaridad al mayor número de personas, a fin de poder disponer del capital humano necesario para ser incorporado en el proceso de desarrollo económico marcado por la industrialización, el crecimiento urbano y la organización del Estado orientado por directrices de planificación y descentralización.

La aproximación histórica al proceso de crecimiento económico de la ciudad de Pereira (1969-1972), permite tanto la caracterización de la educación media, como la del sistema INEM: proyecto modernizador de la media que constituyó no solo una alternativa a la creciente demanda educativa, sino que también, con su currículo flexible y diversificado, aportó, de alguna manera, mano de obra calificada para el mercado laboral, razón de ser del proyecto.

Palabras clave: escuela expansiva, educación media diversificada, historia de la educación media.

\begin{abstract}
The article presents an approach to the modernization's process of the secondary education at Pereira. The INEM System was an expression of the educative modernization lived in the country from the second half of the XX Century. According to Martinez Boom, it was developed in two times: expansive and competitive school. The period between 1950 and 1980
\end{abstract}

\footnotetext{
* El presente artículo respeta las directrices y normas dispuestas en la Declaración de Ética de Publicación de Ciencia Nueva, Revista de Historia y Política. Esta declaración puede consultarse en la página web de la revista: revistas.utp.edu.co/index.php/historia

** Licenciada en Ciencias Sociales de la Universidad Tecnológica de Pereira. Abogada de la Universidad Libre Seccional Pereira. Especialista en Orientación Educativa y Desarrollo Humano de la Universidad del Bosque. Magíster en Historia de la Universidad Tecnológica de Pereira. Docente de la Fundación Universitaria del Área Andina de Pereira.
} 
corresponds to the modernization's first phase (expansive school). It is based on the development model in terms of economic growth, which demands the school expansion. This first phase is understood as the quantitative cycle of the school to extend the scholarship for the maximum number of people, in order to have the necessary human capital to be incorporated in the economic process marked by industrialization, urban growth and state organization leaded by guidelines for planning and decentralization.

The historical approach to the economic growth process of the city of
Pereira (1969-1972) allows both the characterization of the secondary education and the INEM system: a modernizing project of the secondary that was not only an alternative to the growing educational demand, but also, with its flexible and diversified curriculum, contributed, in some way, providing qualified workforce for the labor market, reason for being of the project.

Keywords: expansive school, diversified secondary education, secondary education history.

\section{Introducción}

7 n Colombia desde 1962 la educación media es considerada como el nivel educativo ubicado entre la educación primaria y la educación superior. La educación media como objeto de investigación es un tema poco abordado, menos cuando se habla de educación media diversificada o sistema INEM.

Hacia 1950 la educación secundaria o media se encontraba inserta en un contexto premoderno, caracterizado por ser: "elitista confesional y tradicional"1. Es después de la segunda mitad del siglo XX que se dan una serie de reformas en respuesta a procesos de modernización a nivel económico y social, generadas por el crecimiento económico.

Históricamente "la educación media como sistema organizado para la adquisición y el desarrollo de conocimientos es una institución que nace con el proceso de modernización social"2. La educación media hasta comienzos del XX se caracterizó por una orientación académica e intelectualista, situación coherente con el escaso desarrollo industrial. En otras palabras, "la naturaleza de la división del trabajo, el bajo grado de tecnificación del sistema productivo"3 determinó que la función de la escuela, entendida esta como la institucionalización de la educación primaria y secundaria, no fuera económica en términos de preparar mano de obra; por el contrario, el carácter cerrado y academicista la hizo selectiva en términos de acceso y desprovista de fines económicos.

Es después de la segunda mitad del siglo XX que la educación y el desarrollo económico van a la par. "El desarrollo como estrategia, generada en los países industrializados" $"$ es adoptado por América Latina y en este caso Colombia como camino a seguir. Este enfoque hace necesario que el mundo industrializado asista al mundo atrasado y

\footnotetext{
${ }^{1}$ Germán Rama, Desarrollo y educación en América Latina y el Caribe Tomo II, Capítulo VII (Argentina: UNESCO, CEPAL, PNUD, 1987), 14.

${ }^{2}$ Rama, Desarrollo y educación...,20.

${ }^{3}$ Rodrigo Parra Sandoval, La calidad de la educación. Universidad y cultura popular (Bogotá: Fundación FESTercer Mundo Editores, 1992), 32.

${ }^{4}$ Arturo Escobar, La invención del Tercer Mundo. Construcción y deconstrucción del desarrollo (Bogotá: Fundación Editorial el perro y la rana, 2007), 82.
} 
subdesarrollado en aspectos como la planificación de la economía, la organización y la reestructuración del Estado y sus instituciones (incluyendo la escuela).

De tal forma que el contexto económico nacional orientado hacia la industrialización, ve en la educación la proveedora de mano de obra capacitada para enrolarse en el mundo laboral. Es por ello que hacia 1945 se asume como tarea de Estado respecto de la educación media, la organización y reglamentación de la educación técnica (Ley 0143 de 1948 "Por la cual se organiza la educación técnica); ella constituye el primer paso para enrolarse en el proceso de modernización.

La modernización del Estado y de la educación en sus dos fases, escuela expansiva y competitiva $^{5}$, exigió cambios en sus instituciones (Ministerio de Educación Nacional), al igual que constantes reformas que durante todo el siglo XX se direccionaron no solo a concebir el sistema educativo (Decreto 088 de 1976), sino también a definir y organizar la educación media (Decreto 45 de 1962).

La escuela expansiva obedece a la necesidad de avanzar en la institucionalización de la educación, de alfabetizar a una población sumida en la ignorancia, el atraso y la pobreza. De ahí que la educación sea considerada como la clave del desarrollo y del crecimiento económico. La modernización educativa se articula a procesos de planificación, elaboración de diagnósticos, identificación de recursos y objetivos. De esta manera, la educación significó el inicio de un proceso extensivo e intensivo de escolarización de la población.

Parafraseando a Martínez Boom ${ }^{6}$, esta primera fase es entendida como el ciclo cuantitativo de la escuela. La prioridad de la educación es extender la escolaridad al mayor número de personas a fin poder disponer del capital humano necesario para ser incorporado en el proceso de desarrollo económico, marcado por la industrialización, el crecimiento urbano y la organización del Estado.

La segunda mitad del siglo XX fue amplia en cuanto a reformas educativas. En el contexto de la escuela expansiva, el proyecto diversificado de la educación formalizado a través del Decreto 1962 de 1969 asciende, por un lado, como expresión de la asistencia técnica y financiera internacional y, por otro, como respuesta a la necesidad de cualificar el recurso humano al servicio del naciente proceso de industrialización.

Otras medidas adoptadas dentro del contexto de la escuela expansiva en relación con la educación media, lo constituyen: el fomento de los colegios cooperativos para la educación popular (Ley 9 de 1971), las jornadas adicionales en los planteles educativos (Decreto 2854 de 1974) y la reglamentación sobre el número de estudiantes en los establecimientos oficiales de educación media y elemental, los cuales funcionarán con un máximo de 50 alumnos por curso y un mínimo de 30, en aras de dar mayor oportunidad de educación a la población en edad escolar (Decreto 1997 de 1975).

Para realizar el acercamiento al proceso de modernización de la educación media en Pereira, en primera instancia, el artículo hace una aproximación al contexto histórico de la educación media y el proyecto modernizador o sistema INEM como materialización de la escuela expansiva. Seguidamente, se caracteriza la educación media diversificada. Para finalizar, se recoge el contexto socioeconómico local dentro del cual se implementa la educación media diversificada. La ciudad de Pereira fue una de las diecisiete ciudades en la que el proyecto educativo se desarrolló, evidenciando, de una parte, los cambios en términos

\footnotetext{
5 Alberto Martínez Boom, De la escuela expansiva a la escuela competitiva (Bogotá: Editorial Anthropos, 2004), 13.

${ }^{6}$ Martínez Boom, De la escuela expansiva...,111- 112
} 
de crecimiento socioeconómico y, de otra, la necesidad de ampliar la oferta educativa local como respuesta a las exigencias de la economía en el lapso comprendido entre 1969 y 1972.

\section{Metodología}

El desarrollo de este artículo se realizó a partir de la revisión del trabajo "El INEM y la media diversificada: Un acercamiento a los procesos de modernización de la educación en Pereira 1969 - 1994"

La educación media diversificada fue un proyecto centralizado, que se implementó en diecisiete departamentos de la geografía nacional. Es por ello que además de hacer una aproximación histórica de la educación media y particularmente a la media diversificada, también se caracteriza su implementación en Pereira (contexto local) durante el periodo comprendido entre 1969-1972, lapso que evidencia los cambios socioeconómicos (crecimiento económico, poblacional) y su relación directa con la educación media diversificada. Este proyecto de educación, además de ampliar la oferta educativa, constituye una respuesta a las necesidades económicas del proceso de industrialización y crecimiento económico. Todo ello da cuenta del proceso de modernización de la educación en su fase expansiva, dentro del cual asciende y se consolida la educación media diversificada en la ciudad de Pereira.

El abordaje teórico de la modernización de la educación y la historia de la educación en Colombia se realiza a partir de los trabajos realizados por Alberto Martínez Boom, Rodrigo Parra Sandoval, Aline Helg, Víctor Manuel Gómez Campo, Martha Cecilia Herrera C., Olga Lucía Zuluaga Garcés. Por ser la educación media diversificada un proyecto educativo de orden nacional, centralizado, enmarcado dentro del proceso de modernización el cual se formalizó a través de normas, informes y manuales, se revisaron fuentes primarias como los documentos de orden internacional y nacional que permitieron dimensionar el sistema INEM, igualmente a través de lo departamental y local se reconstruyó el contexto dentro del cual se implementó el sistema INEM.

\section{La educación media en Colombia y el sistema INEM}

Antes de abordar el proceso de modernización de la educación media a través del sistema INEM es pertinente hacer un esbozo histórico de la misma. En América Latina la educación media se ha caracterizado por ser "la última en aparición cronológica en los sistemas educativos nacionales" ${ }^{\text {, }}$ además por una fuerte indefinición respecto de la función social y su estructura organizativa. La educación media en Colombia no es ajena a lo anterior. Por un lado, el acceso selectivo, elitista y de carácter preparatorio para la educación superior y de otro, la función social dual que se debate entre el predominio académico respecto del técnico y tecnológico.

Inicialmente, la estructura organizativa de la educación media pasó por diferentes momentos dado que el Estado estuvo en el proceso continuo de modernización de sus instituciones, entre ellas el Ministerio de Instrucción Pública (1886), el Ministerio de Instrucción y Salubridad Pública (1923) y el Ministerio de Educación Nacional-MEN (1928). La organización administrativa, financiera y pedagógica del MEN hizo parte del programa de los diferentes gobiernos a lo largo del siglo XX.

\footnotetext{
${ }^{7}$ Alba Lucia Pérez Gil, "El INEM y la media diversificada: Un acercamiento a los procesos de modernización de la educación en Pereira 1969 - 1994” (tesis de maestría, Universidad Tecnológica de Pereira, 2016).

${ }^{8}$ Rama, Desarrollo y educación...,14.
} 
A partir de la segunda mitad del siglo Xx el proceso de modernización económica, política y educativa constituyó el escenario de la escuela expansiva. Hacia la década de 1960, la Misión conjunta UNESCO-AID-BIRF promueve su interés por la situación de la educación media y superior. A continuación, su misión:

[...] No interesa solo aumentarla cantidad de educación que se impartirá en el futuro a las promociones nacionales de los distintos niveles, sino de mejorar la calidad de educación que se va a impartir. Todavía más: se trata de cualificar o diversificar la educación (o, si se quiere, la formación), para que de esta manera rinda al aporte que se le exige de aptitud, productividad y eficiencia humanas en la tarea del desarrollo 9 .

Las políticas educativas colombianas han estado ligadas a recomendaciones; asistencia financiera, técnica y organizacional externa. Tal es el caso del proyecto para la educación secundaria o media presentado por el Banco Internacional para la Reconstrucción y el Desarrollo el cual diseña en su totalidad el sistema de educación media diversificada. "Hasta 1970, fuera del SENA y de los INEM, ningún programa de educación primaria o secundaria obtuvo una financiación externa de importancia" ${ }^{10}$.

En la década de los sesenta el gobierno nacional solicitó la colaboración de varias entidades internacionales como la UNESCO, BIRF, AID y se organizó la Misión de Planeamiento para la Educación Nacional de Colombia (1964-1965), dentro de la cual se presentó el informe de Glenn F. Warner, experto del AID, quien realizó un estudio diagnóstico de la educación media y concluía que:

Colombia tiene una gran deficiencia de recursos humanos entrenados, necesarios para el desarrollo político y económico del país, y esta deficiencia tiene su origen en los programas educacionales inadecuados a todos los niveles. El número total de estudiantes que asisten y completan cualquier nivel de la educación es inadecuado para satisfacer en un mínimo las necesidades futuras de recursos humanos para el crecimiento y desarrollo en muchos $\operatorname{campos}^{11}$.

Ante la situación crítica de la educación, el mismo informe "presenta un plan global para mejorar la educación secundaria que se centraba en la creación de las llamadas 'escuelas comprensivas"" . La escuela comprensiva corresponde a un modelo educativo emergente en Norte América, y definido por Warner así:

Una escuela que combina funciones de las escuelas especializadas en un programa unificado. La matrícula grande de una escuela de este tipo hará posible que su funcionamiento sea económico, puesto que los alumnos que estudian materias especializadas se mezclan democráticamente los unos con los otros en el estudio de materias comunes a todas en el currículo, así como en la utilización de todas las facilidades de la escuela ${ }^{13}$.

\footnotetext{
${ }^{9}$ Hernando Gómez Ochoa, Espacios para la Educación Escolar y Extraescolar Caso de Colombia (Santiago de Chile: UNESCO, 1983), http://unesdoc.unesco.org/images/0005/000561/056160SB.pdf.

${ }^{10}$ Yvon Le Bot, Educación e Ideología en Colombia (Medellín: La Carreta Editores, 1979), 62.

${ }^{11}$ George Psacharopoulos, Eduardo Vélez y Antonio Zabala, Una evaluación de la educación media diversificada en Colombia (Bogotá: Rojas Eberhard Editores LTDA, 1986), 22.

${ }^{12}$ Psacharopoulos, Vélez y Zabala, Una Evaluación de la Educación...,22.

${ }^{13}$ Alberto Martínez, Carlos Noguera y Jorge Castro, Modernización y Currículo (Bogotá: Editorial Magisterio, 2013), 147.
} 
El sistema INEM se fundamentó en la escuela comprensiva. Las ventajas de los INEM según el mismo Warner radican en que el nuevo currículo puede servir de patrón para otras escuelas, así como de centro de demostración y preparación para los maestros. Una educación al servicio de las diferencias individuales y de la convivencia democrática, en la medida de que confluyen sectores sociales excluidos de la educación media por su carácter elitista, lo que implica diversidad social y cultural en el estudiantado.

El sistema diversificado a través de la escuela comprensiva también dio respuestas a las necesidades de desarrollo económico; el currículo bivalente, flexible y diverso; permitió tanto el acceso a la educación superior como al mundo del trabajo. El creciente desarrollo industrial y comercial requería de personal medianamente calificado, situación que encuentra justificación en la denominada "teoría del capital humano"14. Máxime por que el acceso a la educación superior era insuficiente y elitista, por ende, la diversificación de la educación media se constituía en un modelo eficiente para las nuevas exigencias sociales y económicas.

La sociedad industrial moderna requiere un alto grado de diversificación y especialización de la estructura ocupacional, en función de la creciente división y especialización del trabajo y los saberes, de tal manera que el grado de diversificación de las profesiones y ocupaciones constituye un importante indicador del desarrollo industrial y científico-tecnológico en determinada sociedad ${ }^{15}$.

La función económica orientada por la diversificación y diferenciación educativa, encuentra en el sistema INEM la respuesta educativa acorde al desarrollo industrial. Un currículo flexible y diverso, que no solo satisfacía las orientaciones en términos de aptitudes del estudiante, sino también lo habilitaba para desempeñarse con determinado grado de cualificación en el mercado laboral. Sobre todo, teniendo en cuenta que, dentro del contexto de la media, a excepción del bachillerato técnico (creado según el Decreto 0884 de 1946), el bachillerato era tradicional, clásico o académico.

El sistema INEM fue un proyecto financiado, diseñado, direccionado y evaluado por agentes externos con base en recomendaciones establecidas por: la UNESCO, el Banco Internacional de Reconstrucción y Fomento-BIRF y la Agencia de los Estados Unidos para el Desarrollo-USAID ${ }^{16}$. El desarrollo del proyecto educativo se realizó en dos fases, como se describe a continuación.

En el período 1963-1966, la UNESCO y la Agencia de los Estados Unidos para el Desarrollo con base en estudios previos habían identificado la necesidad de modernizar y cambiar la educación secundaria por un sistema integral. Posteriormente, se ayudó al gobierno de Carlos Lleras Restrepo en la elaboración del proyecto de reforma de la educación secundaria, el cual consistió en la construcción y equipaje de 19 grandes institutos de educación secundaria.

En julio de 1968, el BIRF aprobó un préstamo de US \$ 7,6 millones (que representaba el $50 \%$ del costo total estimado del proyecto) para ayudar a financiar la primera fase de este programa que constaba de diez de estas escuelas (Préstamo No 522-CO). El Decreto 1962 del 20 de noviembre de 1969 estableció la enseñanza media diversificada y preceptúa que a partir

\footnotetext{
${ }^{14}$ Martínez Boom, De la escuela expansiva...,81.

${ }^{15}$ Víctor Manuel Gómez Campo, La educación media en Colombia: un estudio del modelo INEM (Bogotá: Universidad Nacional, Facultad de Ciencias Humanas, 1995), 19.

${ }^{16}$ Appraisal of Second Education Project Colombia, March 25, 1970, p.2. Traducción libre de Alba lucia Pérez.
} 
de 1970 funcionara en el país, en Barranquilla, Bogotá, Bucaramanga, Cali, Cartagena, Cúcuta, Medellín, Montería, Pasto y Santa Marta.

Las nueve escuelas restantes se aplazaron a una segunda fase con el fin de tener un número suficiente de docentes formados para enseñar el nuevo plan de estudios y para reducir el proyecto a un tamaño más manejable.

En marzo de 1969, el gobierno de Lleras Restrepo presentó una solicitud de financiamiento para la segunda fase del programa, que comprendía la construcción y el equipamiento de las nueve escuelas integrales restantes: Manizales, Pereira, Ibagué, Armenia, Neiva, Popayán, Tunja, Villavicencio y Bogotá (segundo instituto) ${ }^{17}$.

El proyecto contemplaba el establecimiento de un sistema de procesamiento de datos informáticos para la administración, y el uso de un sistema de televisión de circuito cerrado de instrucción para los 19 institutos de secundaria. Puntos que fueron retirados del proyecto inicial.

El proyecto INEM reforzaría la introducción del concepto de escuela integral o comprensiva en la educación secundaria en Colombia. Sería un instrumento valioso para la política del gobierno a fin de preparar a los estudiantes tanto para el mercado de trabajo, como para la educación superior.

Además del concepto de escuela integral o comprensiva, se adelantaría un plan de estudios que integraría asignaturas prácticas y académicas con una educación general adecuada y equilibrada, la cual aumentaría el abanico de posibilidades para la formación y el empleo. Además, la inscripción en cada instituto permitiría la utilización eficiente de las instalaciones y el personal. Por otro lado, la unidad administrativa del Instituto Colombiano de Construcciones Escolares (ICCE) sería la responsable de la supervisión general del proyecto (Decreto 1962 de 1969).

De la misma forma, el proyecto propuesto aceleraría el cambio tan necesario en el enfoque de la educación secundaria (económico), de la rama académica hacia las ramas industriales, comerciales y agrícolas. Lo anterior exigía flexibilidad y diversificación curricular, al igual que la masificación de esta educación, llevándola a un mayor número de habitantes. El sistema INEM permitía todo lo expuesto, constituyéndose en fiel expresión de la modernización educativa en su fase expansiva.

El sistema INEM hizo parte de la planeación educativa, entendida esta como el conjunto de acciones sistematizadas, coordinadas y orientadas hacia la consecución de unos objetivos, exigiendo como resultado la cualificación y diversificación del recurso humano para satisfacer las necesidades de mano de obra del reciente desarrollo industrial. Según Martínez Boom ${ }^{18}$, la planificación educativa significó un proceso intensivo y extensivo de la escolarización de la población, el cual se manifestó a través de la restructuración del MEN, la legislación educativa, la ampliación de la cobertura y la unificación de planes y programas. De manera que se erradicara la diferencia entre la educación urbana y rural. Asimismo, sirvió para introducir el diseño instruccional como modelo curricular.

\footnotetext{
${ }^{17}$ Igualmente, a través del Decreto 1388 de 1970 se determinaron los nombres que llevarían los institutos de educación media diversificada. Los dos de Bogotá: Francisco de Paula Santander y Santiago Pérez. Medellín: José Félix de Restrepo. Cali: Jorge Isaacs. Cúcuta: José Eusebio Caro. Bucaramanga Dámaso zapata. Cartagena: Antonio J. de Irizarri. Barranquilla: Miguel Antonio Caro. Tunja: Carlos Arturo Caro. Tunja: Carlos Arturo torres. Santa Marta: Simón Bolívar. Montería: Lorenzo María Lleras. Pasto: Mariano Ospina Rodríguez. Popayán: Francisco José caldas. Ibagué: Manuel Murillo Toro. Manizales: Baldomero Sanín Cano. Pereira: Felipe Pérez. Armenia: José Celestino Mutis. Neiva: Eustorgio Salgar y Villavicencio: Luis López de Mesa.

${ }^{18}$ Martínez, Noguera y Castro, Modernización y Currículo...,52.
} 
De esta manera, la educación media diversificada se convierte en objeto de reglamentación y organización, en la medida que debe ajustarse a parámetros de productividad y eficiencia en aras del desarrollo económico.

Sin embargo, el proceso de modernización y desarrollo de la educación media fue desigual y selectivo, algo muy a tono con "el proceso de modernización en Colombia que fue de naturaleza desigual en su desarrollo"19. El desarrollo dual marcó la modernización, la industrialización y el crecimiento urbano, el cual se encontró focalizado en determinadas áreas de la geografía nacional, generando concentración poblacional, centralización de oportunidades y de decisiones y recursos. Por consiguiente, la implementación de la instrucción secundaria fue desigual y estuvo anclada al desarrollo urbano. "La tasa de escolarización secundaria era más elevada a medida que el departamento estaba más urbanizado y sus actividades económicas estaban ligadas a los sectores secundarios y terciarios" ${ }^{\prime 20}$, tendencia que se evidencia al relacionar la tasa de escolaridad de la enseñanza secundaria señalada por Helg en 1923 vs. la implementación del sistema INEM 1969 -1972. A excepción del departamento de Meta, en todos los demás departamentos coinciden los niveles de escolaridad con la implementación de la educación media diversificada.

\section{TASA DE ESCOLARIDAD DE LA ENSEÑANZA SECUNDARIA \\ EN 1923 VS. INEM 1969 - 1972}

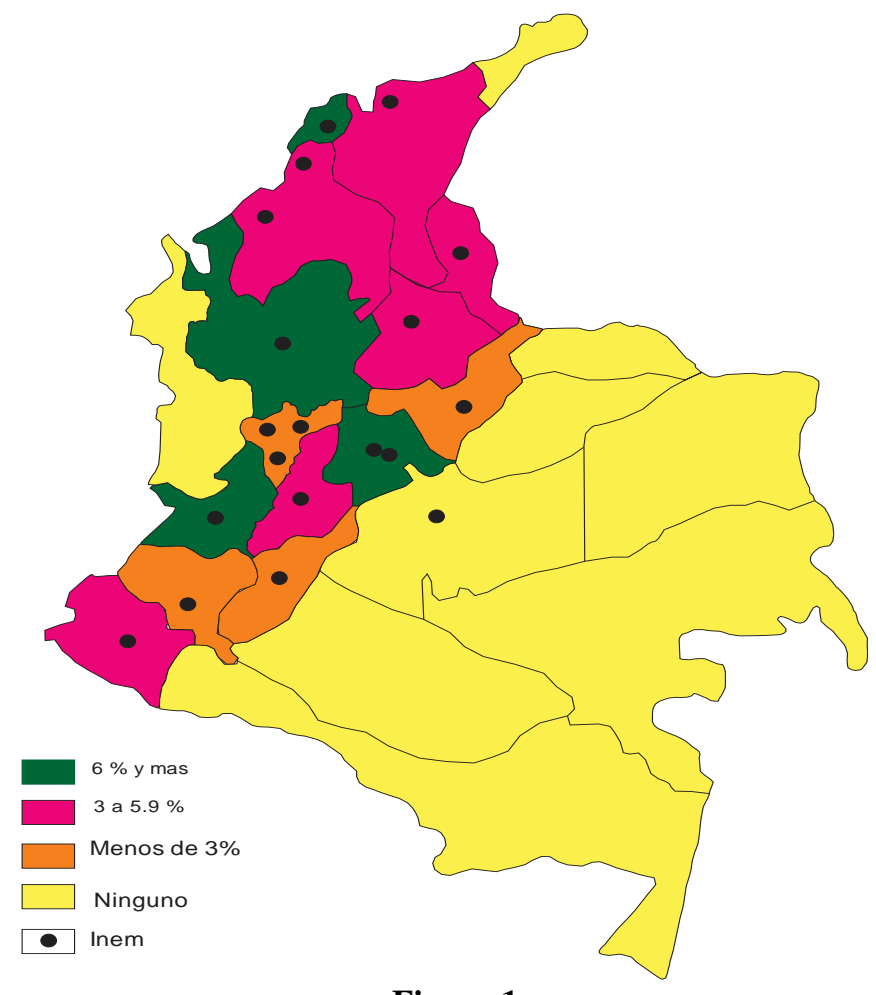

Figura 1.

Tasa de escolaridad de la enseñanza secundaria en 1923 vs 1962-1972.

\footnotetext{
${ }^{19}$ Rodrigo Parra Sandoval, La calidad de la educación...,19.

${ }^{20}$ Aline Helg, La educación en Colombia: una historia social, económica y política (Bogotá: Plaza \& Janés Editorial S.A., 1987),70.
} 


\title{
Desarrollo de la educación media diversificada
}

Un Instituto de Educación Media Diversificada (INEM) es definido como:

[...] aquél que bajo administración unificada ofrece varios programas académicos y vocacionales, tendiente a la obtención del grado de bachiller. En estos institutos el alumno se familiariza primero con disciplinas de educación general y luego escoge entre varias aéreas y modalidades, previamente establecidas ${ }^{21}$.

La implementación de la escuela comprensiva integra diferentes modalidades de bachillerato bajo una misma administración, de acuerdo a las necesidades de cada región. La diversidad en los planes de estudio constituye una de las principales características del sistema INEM.

Inicialmente la educación media diversificada y la educación tecnológica se extiende a los Institutos Técnicos Agrícolas (ITA) por medio del Decreto 1592 de 1974, estableciendo un lapso de siete años lectivos (14 semestres), distribuidos en tres ciclos, así: ciclo básico, ciclo vocacional y ciclo de formación tecnológica. El ciclo básico constaba de cuatro años y comprendía dos etapas, en las cuales, además de lo académico, realizaban actividades de exploración e iniciación vocacional, y un ciclo vocacional de acuerdo a las modalidades de Agropecuaria y Promoción Social.

El Decreto N. ${ }^{\circ} 088$ de 1976 en su artículo 10 formalizó la implementación de la diversificación, extendiéndola a la educación media. Se conserva con el doble propósito de preparar al alumno para los estudios superiores y para el ejercicio laboral en profesiones técnicas y auxiliares. La educación media e intermedia exige la enseñanza diversificada y comprende dos etapas, así:

\begin{abstract}
a. educación media vocacional, que a partir de la educación básica conduce al grado de bachiller. Tendrá una escolaridad de cuatro (4) semestres y se diversificará en modalidades; b. educación intermedia profesional que, a partir del bachillerato y con duración de cuatro (4) semestres, se diversificará en ramas profesionales. Conduce al grado de técnico profesional intermedio en la rama correspondiente.
\end{abstract}

La enseñanza en la educación intermedia profesional será esencialmente práctica y el diploma acreditará para el ejercicio legal de las profesiones técnicas y auxiliares ${ }^{22}$.

Por razones técnicas, administrativas, económicas y de democratización de la educación, se hace necesario establecer centros y mecanismos que permitan la integración de los distintos recursos educativos y su óptima utilización para la extensión de la educación media diversificada. Para ello el Gobierno nacional por medio del Decreto 327 de 1979 creó los ocho Centros Auxiliares de Servicios Docentes (CASD). La construcción y dotación de los mismos fue obra del ICCE, se realizaron en diversas ciudades del país: Florencia, Sabanalarga, Villavicencio, Medellín, Cúcuta, Manizales, Barrancabermeja y Armenia. Los CASD se definen de la siguiente forma:

\footnotetext{
${ }^{21}$ Víctor Manuel Gómez Campo, La educación media en Colombia...,27.

${ }^{22}$ Decreto N. ${ }^{\circ} 088$ de 1976 , artículo 10.
} 
Son unidades administrativas dependientes del Ministerio de Educación Nacional, establecidas con el propósito de ofrecer servicios educativos en el ciclo de educación media vocacional a los planteles que adopten el currículo de educación media diversificado ${ }^{23}$.

Los Centros Auxiliares de Servicios Docentes se constituyeron en centros de apoyo docente, se sujetaron a las normas curriculares contempladas en el Decreto 1419 de 1978. Asignándoles funciones de formación, asesoría técnico pedagógica, coordinación de actividades y capacitación de adultos. Su principal característica fue la extensión del modelo diversificado. Fue otra forma de extensión de la escuela expansiva en cuanto a la educación media.

Los CASD integraron recursos humanos, físicos y económicos con el objeto de proporcionar los medios educativos necesarios para el desarrollo del componente diversificado en los planteles oficiales de educación media clásica de la zona de influencia del centro. Ellos contaban con aulas especializadas, laboratorios, talleres, bibliotecas y ayudas educativas para el desarrollo de las asignaturas prácticas de formación vocacional.

El artículo 51 del Decreto 088 de 1976 estipuló con respecto a los INEM que se convertirían en institutos "de carreras intermedias, con los ciclos de educación media vocacional e intermedia profesional". Disposición que nació muerta, pues nunca se materializó. Por el contrario, la Ley 115 de 1994 desconoció la experiencia e infraestructura del sistema INEM respecto de la organización y orientación de la media, estableciendo la educación media con carácter académico o técnico. De manera que la educación media técnica quedó en situación de dependencia, en la medida que está supeditada a procesos coordinados con el SENA u otras instituciones de capacitación laboral o del sector productivo.

El lapso de consolidación de la educación media diversificada fue formal y muy corto. Este proyecto se materializó en medio del avance de la descentralización administrativa, centralizado en su implementación, dado que era administrado inicialmente por el ICCE a través de la Subgerencia Pedagógica; posteriormente por el MEN a través de la División Especializada de Educación Media Diversificada hasta 1976 cuando se inicia el proceso descentralizador respecto de los INEM; pasando en 1988 la administración financiera a manos del FER y en 1993 al municipio de Pereira.

\section{La educación media en Pereira: contexto socioeconómico 1969-1972}

La economía colombiana a mediados del siglo XX entra en un proceso de expansión industrial "acompañada de la formación de un eje urbano industrial en la zona cafetera (occidente andino)" ${ }^{24}$. Entre las ciudades que comprenden este eje se encuentra Pereira. Algunos factores que explican la formación de este nuevo eje urbano según Cuervo ${ }^{25}$ son el crecimiento extensivo de la producción cafetera, sumado al desarrollo de actividades urbanas de acompañamiento para el mejoramiento de la productividad asociada al café.

\footnotetext{
${ }^{23}$ Gómez, Espacios para la Educación Escolar y Extraescolar...,29.

${ }^{24}$ Mauricio Cuervo y Josefina Jaramillo, Desarrollo económico, urbanización y primacía urbana en Colombia (Bogotá: Tercer mundo Editores, 1997), 314.

${ }^{25}$ Cuervo y Jaramillo, Desarrollo económico, urbanización...,314.
} 
Si bien es cierto que el desarrollo y crecimiento urbano estuvo anclado a la economía cafetera y su acompañamiento, también lo es que la bonanza duró poco ${ }^{26}$. Rápidamente se entró en un proceso de industrialización. Los excedentes de capital en la separación de la producción y la comercialización del café, al igual que la desestructuración de la hacienda cafetera, impulsaron el desarrollo de otras actividades productivas (textil, de alimentos). La presencia de mano de obra calificada no se hizo esperar, fueron pasos firmes en la configuración de la nueva estructura económica del país.

Algunos factores importantes en el proceso de desarrollo y modernización económica y social fueron: el crecimiento y desarrollo de los centros urbanos, la participación del Estado en la dotación de infraestructura vial y de comunicaciones, la modernización institucional, el mejoramiento de los ingresos por las exportaciones y el aumento de la oferta interna por la vía de las importaciones e inmigraciones que permitieron la transferencia de conocimiento técnico.

La modernización demanda cambios de orden sociocultural en procura del crecimiento económico y social, para lo cual se hace necesario el cumplimiento de la función económica de la educación:

El surgimiento de un sistema masivo de educación pública, la incorporación acelerada de tecnologías de comunicación provenientes de los centros económicos avanzados, el cambio de valores sociales y percepciones acerca del trabajo, la riqueza, el empleo del tiempo, la función de la ciencia, etcétera ${ }^{27}$.

La década de 1960 fue muy importante para la ciudad de Pereira. En 1963 sus pobladores celebraban el primer centenario de la fundación oficial, lo que fue motivo de regocijo y esperanza para la urbe. Asimismo, esta efeméride propició la realización de la obra Historia de Pereira que fue financiada por el Club Rotario, en la cual participaron reconocidos académicos como Juan Friede, Luis Duque y Jaime Jaramillo Uribe, en la actualidad este continúa siendo el trabajo más serio acerca de la historia de la urbe. De igual forma, Pereira se convirtió en 1967 en la capital del nuevo departamento de Risaralda, que fue aprobado el 1 de diciembre de 1966.

Ser capital del joven departamento de Risaralda, ayudó a Pereira a insertarse aún más en las dinámicas de la modernización y de crecimiento urbano. Se aprovechó su ubicación geográfica por ser parte del eje oriente-occidente, que va de Bogotá a Buenaventura, lo que le imprimió un gran dinamismo económico a la región.

Entre 1969-1970, las actividades que presentaron mayor inversión empresarial, según la Cámara de Comercio ${ }^{28}$ en Pereira, fueron las comerciales, con una inversión de capital de \$ 652301 700,58. A diferencia de las industriales con una inversión de \$ 307581 810,00 y las agrícolas y ganaderas con \$ 156182602,76 .

El total de empresas inscritas en el Registro Público de Comercio de Pereira fue de 1496, de las cuales el $80 \%$ corresponden a actividades comerciales, proporción que en los siguientes dos años aumentaron, lo que denota además del crecimiento urbano, la concentración del desarrollo industrial y comercial y el descuido de otras actividades

\footnotetext{
${ }^{26}$ José Antonio Ocampo, Joaquín Bernal, Mauricio Avella y María Errazuriz, Historia económica de Colombia. La consolidación del capitalismo moderno (Bogotá: Siglo XXI Editores, 1978), 286.

27 “Algunas consideraciones globales sobre 'modernidad' y 'modernización' en el caso colombiano", Jorge Orlando Melo, acceso el 17 de septiembre de 2018, http://www.jorgeorlandomelo.com/modernidad.htm.

${ }^{28}$ Información construida a partir de los anuarios estadísticos de Risaralda entre los años 1969 y 1970.
} 
económicas asociadas a lo rural (café), que otrora ocuparan un renglón importante en la economía local y nacional.

Se fomentó el empleo a la par con el crecimiento económico en los sectores en su orden terciario y secundario. Según la Cámara de Comercio de la ciudad, la ocupación en enero de 1969 era de 21949 trabajadores en diferentes actividades en la ciudad; en febrero de 1970 era de 23 637, tuvo un aumento del 8\% y su distribución, según sectores económicos, era la siguiente (Tabla 1):

Tabla 1.

Ocupación laboral en Pereira durante 1970.

\begin{tabular}{|l|c|c|}
\hline \multicolumn{1}{|c|}{$\begin{array}{c}\text { Actividad } \\
\text { Económica }\end{array}$} & Ocupación 1970 & \% \\
\hline $\begin{array}{l}\text { Industria y } \\
\text { manufactura }\end{array}$ & 10198 obreros y empleados & 43 \\
\hline Comercio & 4061 trabajadores & 17 \\
\hline $\begin{array}{l}\text { Agricultura y } \\
\text { ganadería }\end{array}$ & 3044 trabajadores & 13 \\
\hline Servicios & 2419 personas & 10 \\
\hline Banca y finanzas & 1592 trabajadores & 7 \\
\hline Construcción & 836 trabajadores & 4 \\
\hline
\end{tabular}

Fuente: información construida a partir de los anuarios estadísticos de Risaralda en los años 1969 y 1970.

La industria, manufactura y comercio constituyen las actividades económicas de mayor demanda laboral, lo que exige cualificación de la mano de obra a nivel técnico y comercial, demandando servicios educativos acorde con las necesidades. El currículo flexible y diversificado que caracteriza el sistema INEM contribuía con el cometido.

La triada: desarrollo económico, crecimiento población urbano y aumento de la demanda educativa, hicieron parte de Pereira entre 1968 y 1970. La Tabla 2 muestra cómo la población urbana y el número de alumnos en secundaria es ascendente.

Tabla 2.

Incremento de alumnos entre 1968 y 1970.

\begin{tabular}{|c|c|c|c|}
\hline Años & Población Urbana & $\begin{array}{c}\text { N. }^{\circ} \\
\text { de alumnos en } \\
\text { secundaria }\end{array}$ & $\begin{array}{c}\text { Alumnos por 1.000 } \\
\text { habitantes }\end{array}$ \\
\hline $\mathbf{1 9 6 8}$ & 177055 & 8694 & 49,1 \\
\hline $\mathbf{1 9 6 9}$ & 185122 & 10287 & 55,5 \\
\hline $\mathbf{1 9 7 0}$ & 193478 & 11979 & 61.9 \\
\hline
\end{tabular}

Fuente: Departamento Administrativo de Planeación Municipal. Compendio Estadístico de Pereira 1970, p. 82.

El desarrollo económico, la oferta de empleo y la demanda educativa, son comprensibles con el crecimiento urbano, el cual ha sido una constante desde mediados de siglo XX. Cada vez más el municipio de Pereira ampliaba el espacio urbano. 
Según el Departamento de Planeación Municipal ${ }^{29}$, hacia 1970, el área del municipio de Pereira era de $658 \mathrm{~km}$, de los cuales el área urbana ocupaba 41,1 km correspondientes al 6,2 \%; el área construida era de 7,2 km, equivalente al 17,5\% del área urbana. El Acuerdo Municipal N. ${ }^{\circ} 5$ de enero 19 de 1973 contempló la nueva delimitación del área urbana en el municipio, la cual había aumentado en $67,8 \mathrm{~km}^{2}$, quedando el área urbana definitiva en 108,9 $\mathrm{km}^{2}$ y representando el $16,6 \%$ del área municipal.

De igual forma, con el crecimiento urbano se dio el demográfico. Una encuesta realizada en 1968 por el Departamento Administrativo de Planeación Municipal, arrojó la cifra de una población urbana proyectada de 193478 habitantes, ocupando 720,1 hectáreas y con una densidad poblacional de $268.6 \mathrm{hab} . / \mathrm{km}^{2}$.

Según la División de Estudios Económicos de Planeación Municipal (1970), después de la segunda mitad del siglo XX se presentó un desplazamiento del campo hacia a ciudad. El continuo crecimiento poblacional en la zona urbana mostró no solo la concentración de la población, sino también, en términos de desarrollo, recursos y posibilidades laborales y educativas, en oposición a una disminución de la población rural. Hacia 1951 la población en el área urbana era de 76262 lo que representaba un 66,1\% y la rural 39080 equivalente a un 33,9\% y en 1974 la urbana ascendió a 236349 y la rural 33541 representando un 87,6 $\%$ y $12 \%$, respectivamente.

Con el crecimiento urbano aumentó la demanda de servicios educativos con respecto a la educación media. Situación que en gran medida se debió a la ampliación de la oferta educativa en primaria, asumida como recomendación internacional y tarea del Estado y de los entes territoriales, y formalizada a través del Plan Quinquenal de Educación Integral contemplada en el Decreto N. ${ }^{\circ} 1710$ de 1963.

Entre 1969-1972, periodo correspondiente a la modernización de la educación con escuela expansiva, se observa una creciente demanda de la educación media oficial según datos de Secretaria de Educación Departamental y la Jefatura de Educación Media de Pereira (Tabla 3).

Tabla 3.

Demanda de la educación media en Pereira entre 1969-1972.

\begin{tabular}{|c|c|c|c|}
\hline Años & Oficial & Privada & Total \\
\hline $\mathbf{1 9 6 9}$ & 7140 & 3147 & 10287 \\
\hline $\mathbf{1 9 7 0}$ & 8358 & 3621 & 11979 \\
\hline $\mathbf{1 9 7 1}$ & 9083 & 3558 & 12641 \\
\hline $\mathbf{1 9 7 2}$ & 9456 & 3414 & 12870 \\
\hline
\end{tabular}

Fuente: información construida a partir de los anuarios estadísticos de Risaralda en los años 1969 y 1972.

Según Planeación Municipal, hacia 1970, la modalidad y sectorización de los nueve establecimientos educativos de media oficial era la siguiente: Plaza de Bolívar (sector 43) el Instituto Femenino (académico). Instituto Técnico Superior (técnico), Barrio San José (sector 28.) Rafael Uribe Uribe (académico), Barrio Providencia y Vergel (sector 23). Deogracias Cardona (académico), Barrio Santa Teresita (sector 16). Normal nacional (pedagógico) Barrio la Paz (sector 29). Vocacional e Industrial, Sector Galería Central (sector 44). La

${ }^{29}$ Departamento Administrativo de Planeación Municipal. Compendio Estadístico de Pereira 1970, p. 6. 
Inmaculada (comercial) Barrio Boston (sector 24). Alfonso Jaramillo, Barrio Cuba (sector 1) Jesús María Ormaza (académico).

Cabe señalar que los doce establecimientos privados de educación tenían la particularidad de ofrecer primaria y secundaria (exceptuando el Lestonnac). Atendían a una población de 3146 estudiantes con el predominio del bachillerato clásico (exceptuando La Enseñanza y los Sagrados Corazones que ofertaban secretariado y comercio, respectivamente. La ubicación de estos establecimientos educativos era la siguiente: Popular modelo (sector 34). La Enseñanza (Compañía de María) y el Gimnasio Pereira, Plaza de Bolívar (sector 43). Inmaculado Corazón de María (Franciscanas), el Instituto los Andes y el Instituto panamericano, Providencia y el Vergel (sector 23). El Sagrado Corazón de Jesús (Bethlemitas) y la Salle, Galería Central (sector 44). Nuestra Señora de la Consolación, Camilo Mejía Duque (sector 19). Los Sagrados Corazones, Kennedy (sector 37). Lestonnac, Álamos (sector 27). El Liceo Pereira, el Calasanz, el Jardín (sector 47).

Igualmente existió educación comercial ofertada por instituciones privadas que se asimilan a escuelas comerciales, las cuales debían brindar cuatro años de educación según lo estipulaba el Decreto 2433 de 1945. Su ubicación local correspondía a los siguientes sectores: Comercial Greeg, Lisandrino Cardona, Idesco y Escontal se encontraban ubicados en el sector 44 (Antigua Galería Central) y Colombiana de Comercio en el sector 42 (Lago Uribe). Todos ellos ubicados en sectores que por esa época habían iniciado un proceso de descomposición social, por el desplazamiento de la clase alta hacia otros sectores de la ciudad (Álamos, Alpes, el Jardín).

En síntesis, la educación media oficial y privada se encontraba concentrada en trece sectores de la ciudad, dejando de lado un vasto número de los mismos dado el constante crecimiento urbano y poblacional. Es pertinente señalar que según el Departamento Administrativo de Planeación, hacia 1970, existían 52 sectores en el municipio. El total de la población urbana correspondiente a esos sectores ascendía a 193 478. De acuerdo a la encuesta realizada en 1968, la población proyectada muestra que los sectores de mayor concentración poblacional (superior a los 5000 habitantes) son en su orden: sector Lago Uribe 24027 habitantes, barrio Cuba 15 446, sector Plaza de Ferias 10 389, barrio Corocito con 9547, sector Parque la Libertad 9512, barrio san Jorge 8688, sector Plaza Bolívar 7215, barrio Villavicencio 6944 y barrio Alfonso López 5548.

En cuanto al carácter de la educación local, el 34,6 \% correspondía a los establecimientos urbanos públicos y el $65,4 \%$ a los privados, estos últimos ofertaban enseñanza primaria y secundaria, y en su mayoría correspondían a congregaciones religiosas, lo que le daba un carácter elitista y selectivo a la educación media, en la medida que no toda persona podía ingresar.

Respecto del financiamiento de la educación media oficial en Pereira y durante el mismo año, el presupuesto municipal para la educación media era de \$ 441000 , a diferencia del presupuesto para la educación primaria que era de \$1 183 000. Lo que denota la debilidad presupuestal respecto de la educación secundaria oficial, repercutiendo tanto en la cobertura como en el tipo de bachillerato que se ofertaba.

Es importante señalar que como expresión del crecimiento urbano y de las demandas sociales en 1970 el Instituto de Crédito Territorial (ICT), seccional Pereira, ofertó la construcción de 180 casas en la denominada urbanización el Jardín ${ }^{30}$, lugar en el cual se construyó el INEM. La ubicación resultó siendo estratégica, ya que, al no tener comunidad

${ }^{30}$ El Diario, marzo 18 de 1970. Edición 12494, p.1. 
propia, desde su inicio, los estudiantes que accedieron a la educación diversificada procedían de diferentes barrios de Pereira y Dosquebradas atraídos fundamentalmente por la diversificación. En palabras del jefe de unidad docente del IMEN Hernando Tafur (19742002): "Estaban motivados por la oferta diversificada y posiblemente el interés de los padres por otro tipo de oferta, diferente a la tradicional, que solo, preparaba al estudiante para la Universidad. Los INEM eran instituciones con una dotación amplia y moderna y con una planta física diferente y atractiva".

Por lo anteriormente expuesto, el balance respecto de la educación media oficial en Pereira es el siguiente:

a. En primera instancia, la oferta educativa en la media conservaba en buena medida las características de la educación de comienzos de siglo XX: comunidades religiosas como pioneras de la educación, descuido del Estado en el financiamiento de la educación pública y currículos tradicionales y academicistas.

b. Entre 1969-1972, periodo correspondiente a la escuela expansiva, se observó una creciente demanda de educación media. Según datos de la Secretaria de Educación Departamental y la Jefatura de Educación Media, la población matriculada en secundaria en Pereira, oficial y privada, aumentó: en 1969 el número de estudiantes era de 10 287; en 1970, 11 979; en 1971, 12641 y en 1972 correspondía a 12870.

c. Por otra parte, el sector educativo municipal público y privado reflejaban la hegemonía del bachillerato clásico. El $90 \%$ de la oferta educativa local urbana correspondía al bachillerato académico.

d. De otra parte, hubo diversidad en cuanto al manejo y administración de recursos físicos y humanos, de regímenes laborales y de administración docente. Al igual que hubo desigualdad en la formación docente. Según la Jefatura de Educación Media, en 1971, la distribución del personal docente era de 143 nacionales, 209 departamentales, 57 municipales y 325 privados, para un total de 734; de los cuales 154 estaban en la categoría 1, 92 en la categoría 2, 58 en la categoría 3, 32 en la categoría 4 y 398 sin categoría. Los establecimientos urbanos de educación media en 1971 se agrupaban así: 3 nacionales urbanos, 9 departamentales urbanos y 13 privados.

El proceso de modernización de la educación media a través de la introducción del sistema INEM inicia su periplo con un conjunto de características administrativas y curriculares y en cuanto a presupuesto e infraestructura, que lo hacían en ese momento histórico innovador y moderno respecto de lo instalado en materia educativa.

Las características de los Institutos Nacionales de Educación Media (INEM), se enmarcan dentro del proceso de modernización del Estado y el instrumento técnico ordenador y organizador denominado planeación económica y social; y se pueden agrupar así: administrativas y curriculares.

Las administrativas se enmarcan dentro de la planeación educativa, considerada como el camino para asegurar el logro de los objetivos propuestos, para lo cual comprende diferentes etapas: planeamiento, organización, selección de recursos, dirección, coordinación, información y financiación.

En cuanto a las curriculares según el MEN $1969^{31}$, el currículo de un INEM se caracterizaba por:

\footnotetext{
${ }^{31}$ Ministerio de Educación Nacional. Educación media colombiana. Primera Parte (Bogotá D.E., 1969).
} 
a. Flexibilidad en la organización, por cuanto ofrece al alumno diversas posibilidades para orientar sus estudios.

b. Formación integral, por cuanto propende por el desarrollo armónico del educando.

c. Atención a las diferencias individuales, por cuanto incluye orientaciones didácticas acordes al desenvolvimiento del alumnado.

d. Factibilidad, por cuanto en la planeación se previeron los recursos humanos y materiales necesarios para su desarrollo, al igual que sistemas y procesos de evaluación adecuados al mismo.

El plan de estudios del sistema INEM se desarrollaba a través de tres etapas:

a. Etapa de exploración vocacional: comprendía los dos primeros años. Su objetivo era la adquisición de conocimientos y experiencias en las diferentes actividades ofrecidas en el mercado de trabajo.

A la par con las asignaturas del núcleo común, el alumno rotaba por los cuatro talleres básicos propios de cada área, la rotación se hacía por semestres; terminada esta etapa el alumno con la asesoría de orientación escolar, profesores y padres de familia elegía de acuerdo a sus aptitudes, intereses y necesidades, la rama en la cual deseaba continuar sus estudios a partir de tercero de bachillerato, podía seleccionar entre: académica, industrial, comercial, agropecuaria y promoción social.

b. Etapa de orientación vocacional: comprendía los grados tercero y cuarto de bachillerato. Su objetivo era informar y orientar al alumno en las diferentes actividades que comprendía el área vocacional elegida. Al final de esta etapa el alumno escogía la modalidad del área que más se adecuaba a sus condiciones personales para cursarlas a partir de grado quinto. Las modalidades o tendencias ofertadas eran: humanidades, ciencias y matemáticas, metalmecánica, electricidad y electrónica, construcciones, química industrial, agropecuaria, secretariado, contabilidad, salud, organización comunidad y hogar.

c. Etapa de orientación profesional o educación ocupacional: comprendía los grados quinto y sexto de bachillerato, durante la cual el alumno desarrollaba las posibilidades ocupacionales y educacionales. El título otorgado era el de bachiller, el cual permitía seguir estudios superiores y un certificado de competencia en la modalidad vocacional escogida.

Según Hernando Tafur (directivo 1974-2002), el INEM Felipe Pérez de Pereira ofrecía bachillerato académico con dos modalidades: ciencias y humanidades. Bachillerato vocacional con las modalidades: industrial en construcciones y electricidad. Comercial con secretariado y contabilidad. Bachillerato en promoción social y bachillerato en agropecuaria. El sistema INEM en el municipio fue ganando espacio en poco tiempo, al punto que llegó a realizar selección del personal docente a través del examen de admisión. En palabras de la docente de la institución Sonia Velázquez Uribe (1972-2016):

El proceso de selección de los estudiantes al colegio, al principio, prácticamente era ninguno. Posteriormente, cuando la demanda de cupos creció considerablemente, se aplicaron un examen de admisión y entrevistas. Además, las notas o calificaciones del año anterior, la edad, la nota de comportamiento social (llamada en esa época: “conducta"), preferiblemente que no fuera repitente, entre otros criterios [...] Estos estudiantes empezaron a ser 
privilegiados porque gozaron de muchos beneficios que en otros colegios no existían. Por ejemplo, recibían gratuitamente guías de aprendizaje o de contenido y guías de trabajo de todas las asignaturas. Por lo tanto, no tenía que comprar libros, gozaban de servicios especiales como: médico, enfermería, odontólogo, sicólogos (psicoorientadores), un director de bienestar estudiantil, las mejores canchas deportivas, laboratorios de física, química e idiomas muy bien dotados, salas de bilingüismo, biblioteca, salas de audiovisuales, ayudas educativas y talleres ${ }^{32}$.

Respecto al grado de aceptación y la percepción del INEM en la ciudad, la egresada de la modalidad de Promoción Social (1978) y actual docente de la institución, María Esnelia Vallejo, expresa:

La presencia de esta nueva institución en la ciudad causó mucho impacto, personalmente me sentí muy motivada al igual que muchas niñas de la escuela en la que hice mi primaria, ya que fuimos nosotras quienes llevamos la información a las casas; puesto que el INEM se dio a la tarea de hacerse conocer visitando las escuelas. Acudimos a la convocatoria de inmediato. Llamaba mucho la atención su estructura física, ya que los llamados pertenecíamos, en general, a los barrios más pobres de la ciudad ${ }^{33}$.

De ahí que la ampliación de la oferta educativa no se hizo esperar, ejemplo de ello es que en marzo de 1972 en palabras de la docente Sonia Velázquez Uribe:

Se empezó una especie de brigada por algunos barrios de estrato socioeconómico bajo, haciendo la labor de invitación y convencimiento "puerta a puerta" y fue así como lograron un total de 334 estudiantes matriculados en el primer grado de secundaria, que en esa época se llamaba "nivel I" 34 .

Dos años más tarde, en 1974, el INEM de Pereira contaba con un total de 1072 alumnos distribuidos en 26 secciones $^{35}$.

Independiente del carácter modernizador del sistema INEM, del grado de aceptación dentro de los sectores populares y de la ampliación de la oferta educativa, la proyección de la educación media diversificada no permeó la educación media. Hacia 1979 y 1980 la educación media local seguía siendo profundamente académica, solo que ahora la masificación de la misma exigía la maximización de la infraestructura, de ahí la existencia de colegios con dos y tres jornadas como lo ilustra la Tabla 4.

\footnotetext{
${ }^{32}$ Entrevista personal.

${ }^{33}$ Entrevista personal.

${ }^{34}$ Entrevista personal.

${ }^{35}$ Revista INEM Pereira-1974.
} 
Tabla 4.

Planteles de educación media en el área urbana oficial, según modalidad y carácter. Pereira, 1979.

\begin{tabular}{|c|c|c|c|}
\hline Colegio & Modalidad & Jornada & Carácter \\
\hline Normal Nacional Mixta & Pedagógica & Ordinaria & Nacional \\
\hline $\begin{array}{c}\text { Instituto Técnico Superior } \\
\text { Diurno }\end{array}$ & Industrial & Mañana y tarde & Nacional \\
\hline $\begin{array}{c}\text { Deogracias Cardona } \\
\text { INEM Felipe Pérez }\end{array}$ & Académico & $\begin{array}{c}\text { Mañana, tarde y } \\
\text { noche }\end{array}$ & Nacional \\
\hline Instituto Femenino & Académico & Mañana y tarde & Departamental \\
\hline La Inmaculada & Comercial & Ordinaria & Departamental \\
\hline $\begin{array}{c}\text { Instituto Técnico Superior } \\
\text { Nocturno }\end{array}$ & Académico & Nocturno & Nacional \\
\hline Rafael Uribe Uribe & Académico & Tarde & Departamental \\
\hline
\end{tabular}

Fuente: Departamento Administrativo de Planeación Departamental de Risaralda. Compendio Estadístico. Pereira, 1979.

En últimas, la ampliación de la oferta educativa en la media y la media diversificada hacen parte de la primera modernización en su fase expansiva. La particularidad del sistema INEM es la diversificación y flexibilidad de su currículo, el cual se adecua no solo a los intereses del educando, sino también a las necesidades económicas del contexto.

Expuesto el carácter modernizador de la educación diversificada y su función económica en aras de satisfacer las necesidades de mano de obra cualificada, es válido señalar que queda desbordada al no haber la pujanza industrial necesaria para ser absorbida, en palabras de Parra Sandoval ${ }^{36}$, la función económica de la educación se concentra en los niveles secundario y superior, constituyendo un cuello de botella en términos de oferta versus demanda laboral.

Como se observa en la Tabla 5, la ampliación de la jornada en los diferentes planteles de educación media en Pereira en 1979, área urbana, oficial, ponen de manifiesto el carácter expansivo en términos de cobertura, característica de la primera modernización de la educación media.

Tabla 5.

Ampliación de la jornada en varios colegios. Pereira, 1979.

\begin{tabular}{|c|c|c|c|}
\hline Colegio & Modalidad & Jornada & Carácter \\
\hline Normal Nacional Mixta & Pedagógica & Ordinaria & Nacional \\
\hline $\begin{array}{c}\text { Instituto Técnico Superior } \\
\text { Diurno }\end{array}$ & Industrial & $\begin{array}{c}\text { Mañana y } \\
\text { tarde }\end{array}$ & Nacional \\
\hline Deogracias Cardona & Académico & $\begin{array}{c}\text { Mañana, tarde, } \\
\text { noche }\end{array}$ & Nacional \\
\hline
\end{tabular}

${ }^{36}$ Rodrigo Parra Sandoval. La calidad de la educación..., 33. 


\begin{tabular}{|c|c|c|c|}
\hline INEM Felipe Pérez & Diversificado & $\begin{array}{c}\text { Mañana y } \\
\text { tarde }\end{array}$ & Nacional \\
\hline $\begin{array}{c}\text { Instituto Femenino } \mathbf{1}^{\circ} \mathbf{y} \mathbf{2}^{\circ} \\
\text { Jornada }\end{array}$ & Académico & $\begin{array}{c}\text { Mañana y } \\
\text { tarde }\end{array}$ & Departamental \\
\hline $\begin{array}{c}\text { La Inmaculada } \\
\begin{array}{c}\text { Instituto Técnico Superior } \\
\text { Nocturno }\end{array}\end{array}$ & Comercial & Ordinaria & Departamental \\
\hline Rafael Uribe Uribe & Académico & Nocturno & Nacional \\
\hline
\end{tabular}

Fuente: Secretaria de Educación Municipal. Compendio Estadístico. Departamento Administrativo de Planeación Departamental. Pereira, 1969.

Igualmente, a nivel departamental, hacia 1979, se evidencia que el sector educativo conservaba la prevalencia del bachillerato clásico. La poca incidencia de la educación media diversificada, obedece a que la demanda de la función económica de la educación no fue requerida, dado el bajo índice de diversificación de la economía. La ampliación de la oferta educativa en la media, producto de la escuela expansiva, conservó el carácter académico (Tabla 6). La diversificación como respuesta a las necesidades del desarrollo económico se limitó al desarrollo local. No obstante, hay que resaltar a Santa Rosa de Cabal, que después de Pereira, fue la más diligente con el modelo diversificado.

Tabla 6.

Establecimientos y alumnos matriculados según modalidades en el departamento de Risaralda, 1980.

\begin{tabular}{|c|c|c|c|c|c|c|}
\hline \multirow{2}{*}{ Modalidad } & \multicolumn{3}{|c|}{ Establecimientos } & \multicolumn{3}{c|}{ Alumnos matriculados } \\
\cline { 2 - 7 } & Total & Oficial & Privado & Total & Oficial & Privado \\
\hline Total & 88 & 70 & 18 & 32670 & 26212 & 6458 \\
\hline Académica & 59 & 45 & 14 & 25036 & 20090 & 4946 \\
\hline Pedagógico & 4 & 4 & - & 598 & 598 & - \\
\hline Industrial & 3 & 3 & - & 1728 & 1728 & - \\
\hline Comercial & 11 & 7 & 4 & 3474 & 1962 & 1512 \\
\hline Agropecuario & 10 & 10 & - & 1449 & 1449 & - \\
\hline $\begin{array}{c}\text { Promoción } \\
\text { Social }\end{array}$ & 1 & 1 & - & 331 & 331 & - \\
\hline
\end{tabular}

Fuente: departamento de Risaralda, Oficina de Planeamiento Educativo 1979-1980.

\section{Consideraciones finales}

En las líneas precedentes se expuso el contexto en el cual asciende la educación media diversificada o sistema INEM, el cual se implementa en el marco histórico de la modernización del país, después de la segunda mitad de siglo XX. Este proceso histórico fue jalonado por fuerzas externas orientadas por organismos internacionales (UNESCO, ORELAC, CEPAL, BID), que con asistencia técnica y financiera direccionaron la 
modernización de una sociedad premoderna anclada en la pobreza, el analfabetismo, y el confesionalismo de una educación secundaria academicista, elitista y tradicional.

La educación media diversificada constituyó una propuesta educativa desarrollada en la primera fase de la modernización o escuela expansiva. Se fundamentó en la teoría del capital humano y la escuela comprensiva. Constituyó un proyecto modernizador, exógeno, centralizado en lo administrativo, financiero y pedagógico, de alto contenido social en la medida en que contribuía a mitigar la brecha de la inequidad social. Igualmente, cumplió una función económica en procura de la formación del capital humano necesario para la creciente industrialización.

Pereira constituyó una de las dieciocho ciudades en las cuales se implementó el proyecto de educación diversificada o sistema INEM. El creciente desarrollo económico y social durante la década del setenta la convirtieron en el escenario de la planeación educativa, entendida según Martínez Boom ${ }^{37}$, como el inicio de un proceso intensivo y extensivo de la escolarización de la población que se manifestó en la restructuración del Ministerio de Educación Nacional, la legislación educativa, la ampliación de la cobertura, la unificación de planes y programas, erradicando la diferencia entre la educación urbana y rural. Asimismo, sirvió para introducir el diseño instruccional como modelo curricular.

A pesar del carácter modernizador de la educación diversificada respecto del predominio del bachillerato académico o clásico, su impacto fue limitado. Por una parte, el desarrollo económico como dinamizador de la misma, terminó por circunscribirla a las necesidades propias del desarrollo comercial e industrial del departamento de Risaralda, que al quedarse concentrado en la capital (Pereira) tuvo poca incidencia en los demás municipios, salvo una limitada presencia en Santa Rosa de Cabal. Es decir, el contexto económico marcado por un desarrollo económico dual tanto a nivel nacional como local, determinó en buena medida el alcance de la educación diversificada. Por otra parte, al poner en paralelo un sistema de educación con visos de modernización con un currículo flexible centrado en el estudiante y una pedagogía activa, frente a otro academicista y tradicional, este último terminó por conservar su hegemonía que otrora lo había caracterizado.

\footnotetext{
${ }^{37}$ Martínez Boom, De la escuela expansiva...,52.
} 


\section{Bibliografía}

\section{Fuentes primarias}

Anuarios estadísticos de Risaralda 1969-1998.

Appraisal of Second Education Project Colombia, March 25, 1970.

Compendio estadístico de Pereira 1970-1974.

El Diario, periódico 1969-1970.

Estadísticas educativas del departamento de Risaralda 1976 - 1980.

Ministerio de Educación Nacional. Educación media colombiana. Primera Parte. Bogotá D.E., 1969.

Instituto Colombiano de Construcciones Escolares ICCE, Subgerencia Pedagógica. Evaluación de aprendizaje. Pereira. Septiembre 11 de 1974.

- División especial de enseñanza media grupo de administración. Manual de administración de los INEM, Bogotá D. E. 1980.

Revista INEM Pereira-1974.

\section{Fuentes secundarias}

Cuervo, Mauricio y Josefina Jaramillo. Desarrollo económico, urbanización y primacía urbana en Colombia. Bogotá: Tercer mundo Editores, 1997.

Escobar, Arturo. La invención del Tercer Mundo. Construcción y deconstrucción del desarrollo. Bogotá: Fundación Editorial el perro y la rana, 2007.

Gómez Ochoa, Hernando. Espacios para la Educación Escolar y Extraescolar Caso de Colombia. Santiago de Chile: 1983. http://unesdoc.unesco.org/images/0005/000561/056160SB.pdf.

Gómez Campo, Víctor Manuel. La educación media en Colombia: un estudio del modelo INEM. Bogotá: Universidad Nacional, Facultad de Ciencias Humanas, 1995.

Helg, Aline. La educación en Colombia: una historia social, económica y política. Bogotá: Plaza \& Janés Editorial S.A., 1987.

"International Bank for Reconstruction and Development International Development Association. Appraisal of a Second Education Project Colombia”. Acceso el 17 de septiembre

de

2018. 
http://documentos.bancomundial.org/curated/es/1970/03/722508/colombia-secondeducation-project

Le Bot, Yvon. Educación e Ideología en Colombia. Medellín: La Carreta Editores, 1979.

Martínez Boom, Alberto. De la escuela expansiva a la escuela competitiva. Bogotá: Editorial Anthropos, 2004.

Martínez, Alberto, Carlos Noguera y Jorge Castro. Modernización y Currículo. Bogotá: Editorial Magisterio, 2013.

Melo, Jorge Orlando. "Algunas consideraciones globales sobre 'modernidad' y 'modernización' en el caso colombiano". Acceso el 17 de septiembre de 2018. http://www.jorgeorlandomelo.com/modernidad.htm.

Ocampo, José Antonio, Joaquín Bernal, Mauricio Avella y María Errazuriz. Historia económica de Colombia. La consolidación del capitalismo moderno. Bogotá: Siglo XXI Editores, 1978.

Parra Sandoval, Rodrigo. La calidad de la educación. Universidad y cultura popular. Bogotá: Fundación FES-Tercer Mundo Editores, 1992.

Siglo del Hombre, 1998.

. El tiempo mestizo: escuela y modernidad en Colombia. Bogotá:

Pérez Gil, Alba Lucia. "El INEM y la media diversificada: Un acercamiento a los procesos de modernización de la educación en Pereira 1969 - 1994". Tesis de maestría. Universidad Tecnológica de Pereira, 2016.

Psacharopoulos, George, Eduardo Vélez y Antonio Zabala. Una evaluación de la educación media diversificada en Colombia. Bogotá: Rojas Eberhard Editores LTDA, 1986.

Rama, Germán. Desarrollo y educación en América Latina y el Caribe Tomo II, Capítulo VII. Argentina: UNESCO, CEPAL, PNUD, 1987.

UNESCO. "La Conferencia Sobre Educación Desarrollo Económico y Social en América Latina”. En Proyecto Principal de Educación. Santiago de Chile 5 al 19 marzo de 1962. http://unesdoc.unesco.org/images/0019/001901/190112so.pdf

Zuluaga Garcés, Olga Lucia y Gabriela Ossenbach Sauter. Modernización de los Sistemas Educativos Iberoamerícanos siglo XX. Tomo II. Bogotá: Editorial Magisterio, 1998. 